\title{
Crystal structure of $\delta-\mathrm{Tm}_{2} \mathrm{~S}_{3}$
}

\author{
Joanna KONCZYK ${ }^{1}$, Pavlo DEMCHENKO ${ }^{2}$, Oksana BODAK ${ }^{1,2}$, Grygorii DEMCHENKO $^{2}$, \\ Bernard MARCINIAK ${ }^{1}$, Wojtek PROCHWICZ ${ }^{1}$, Lilija MURATOVA ${ }^{3}$ \\ ${ }^{1}$ Institute of Chemistry and Environment Protection, Jan Dlugosz University, \\ al. Armii Krajowej 13/15, 42-200 Czestochowa, Poland \\ ${ }^{2}$ Department of Inorganic Chemistry, Ivan Franko National University of Lviv, \\ Kyryla i Mefodiya St. 6, 79005 Lviv, Ukraine \\ ${ }^{3}$ Department of Chemistry, Lviv Medical Training University, Pekarska St. 32, 79005 Lviv, Ukraine \\ * Corresponding author. Tel.: +38 0322394 506; e-mail: demchenko@franko.lviv.ua
}

Received December 21, 2007; accepted February 17, 2008; available on-line March 31, 2008

The crystal structure of the $\delta$-modification of thulium sesquisulfide, $\delta$ - $\mathrm{Tm}_{2} \mathrm{~S}_{3}$, has been determined by $\mathrm{X}$-ray analysis of a single crystal (automatic diffractometer Xcalibur3 ${ }^{\mathrm{TM}} \mathrm{CCD}, 1425$ unique reflections, $\mathrm{R} 1=0.0425$, $w R 2=0.0834)$. The compound crystallizes in the $\delta-\mathrm{Ho}_{2} \mathrm{~S}_{3}$ structure type: space group $P 2_{1} / \mathrm{m}, \mathrm{mP30}$, with lattice parameters $a=10.0626(14), b=3.9675(5), c=17.396(2) \AA, \beta=98.655(11)^{\circ}$. The coordination numbers of the Tm atoms are 6 (distorted octahedron) and 7 (mono-capped trigonal prism), whereas the $S$ atoms have coordination numbers 4 and 5 (trigonal and tetragonal pyramids).

Rare-earth alloys and compounds / Crystal structure / X-ray diffraction

\section{Introduction}

The search for novel inorganic compounds is necessary for the development of new materials, and the accurate and precise determination of their crystal structures is the basic requirement for a better understanding of their physical properties. The rareearth metal chalcogenides have attracted large attention because of their interesting and useful physical properties, such as optical, thermoelectric, magnetic and superconducting. Extensive reviews on rare-earth metal chalcogenides can be found in [1,2].

During our systematic investigation of the ternary Tm-Ni-S system with the purpose to synthesize new phases we obtained as by-product several single crystals of $\mathrm{Tm}_{2} \mathrm{~S}_{3}$, suitable for X-ray diffraction studies. The preliminary investigation of the crystal structure showed the isotypism with monoclinic $\mathrm{Ho}_{2} \mathrm{~S}_{3}$ [3]. The existence of a monoclinic $\delta$-modification of $\mathrm{Tm}_{2} \mathrm{~S}_{3}$ was firstly reported in [4], and further confirmed in [5], but only the cell parameters were determined and isotypism with $\mathrm{Ho}_{2} \mathrm{~S}_{3}$ stated. On the contrary, complete structure refinements have been performed on the isotypic compounds $\mathrm{Y}_{2} \mathrm{~S}_{3}, \mathrm{Er}_{2} \mathrm{~S}_{3}$ and $\mathrm{Yb}_{2} \mathrm{~S}_{3}$, even if the latter was refined in the subgroup $P 2_{1}$.

Like the other rare-earth sesquisulfides, $\mathrm{Tm}_{2} \mathrm{~S}_{3}$ is known to adopt several structural modifications, several of them forming at high pressures. Table 1 summarizes the seven modifications of thulium sesquisulfide known up to date. Only for three of them the conditions of formation are more or less well established at the present time.

The aim of this work was the complete determination of the crystal structure of the $\delta$ modification of $\mathrm{Tm}_{2} \mathrm{~S}_{3}$ from good quality singlecrystal X-ray diffraction data.

\section{Experimental}

Needle-shaped single crystals were extracted from a hot-sintered sample of composition $\mathrm{Tm}_{25} \mathrm{Ni}_{25} \mathrm{~S}_{50}$ that had been melted under an argon atmosphere (Ti-getter) in an arc furnace with a water-cooled copper hearth. The single crystal suitable for X-ray analysis was at a preliminary step investigated by the Laue and Weissenberg methods (RKV-86 and RGNS2 chambers, MoK $\alpha$-radiation), then transferred to an

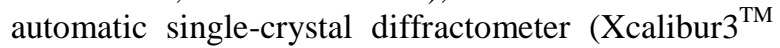
$\mathrm{CCD}$, MoK $\alpha$-radiation, graphite monochromator, $\omega$-scans). The data collection and reduction were performed using CrysAlis CCD [13] and CrysAlis RED [14] programs. The structure was solved and refined with programs from the SHELXL-97 [15] package. The program Structure Tidy [16] was applied to standardize the raw crystallographic data. Additional information about the experimental conditions and some structural parameters can be found in Table 2 . 
J Kończyk et al., Crystal structure of $\delta-\mathrm{Tm}_{2} \mathrm{~S}_{3}$

Table 1 Literature data on thulium sesquisulfides.

\begin{tabular}{|c|c|c|c|c|c|}
\hline \multirow{2}{*}{$\begin{array}{l}\text { Phase, conditions of } \\
\text { formation }\end{array}$} & \multirow{2}{*}{$\begin{array}{c}\text { Pearson symbol, } \\
\text { space group, prototype }\end{array}$} & \multicolumn{3}{|c|}{ Lattice parameters, $\AA$} & \multirow[t]{2}{*}{ Reference } \\
\hline & & $a$ & $b$ & $c$ & \\
\hline $\mathrm{Tm}_{2} \mathrm{~S}_{3}$ & $h R 30, R \overline{3} c, \mathrm{Al}_{2} \mathrm{O}_{3}$ & $6.768(2)$ & & $18.236(3)$ & {$[6]$} \\
\hline $\mathrm{Tm}_{2} \mathrm{~S}_{3}$ & $h P 30, P 6_{3} c m, \mathrm{Yb}_{2} \mathrm{~S}_{3}$ & 6.777 & & 18.32 & [7] \\
\hline $\mathrm{Tm}_{2} \mathrm{~S}_{3}(\theta)$ & $c I 80, \mathrm{Ia} \overline{3}, \mathrm{Mn}_{2} \mathrm{O}_{3}$ & $12.489(4)$ & & & [8] \\
\hline $\begin{array}{l}\mathrm{Tm}_{2} \mathrm{~S}_{3}(\gamma) \\
1900 \mathrm{~K}, 1.2 \mathrm{GPa}\end{array}$ & $c I 28, I \overline{4} 3 d, \mathrm{Th}_{3} \mathrm{P}_{4}$ & $8.223(1)$ & & & [9] \\
\hline $\begin{array}{l}\mathrm{Tm}_{2} \mathrm{~S}_{3} \\
1073 \mathrm{~K}, 2.0 \mathrm{GPa}\end{array}$ & $o P 20$, Pnma, $\mathrm{U}_{2} \mathrm{~S}_{3}$ & $10.479(5)$ & $3.805(3)$ & $10.353(5)$ & 10 \\
\hline $\mathrm{Tm}_{2} \mathrm{~S}_{3}(\delta)$ & $m P 30, P 2_{1} / m, \mathrm{Ho}_{2} \mathrm{~S}_{3}$ & 10.039 & $\begin{array}{l}3.960 \\
\beta=98.78^{\circ}\end{array}$ & 17.363 & [4] \\
\hline & & $10.037(2)$ & $\begin{array}{l}3.954(1) \\
\beta=98.68(2)\end{array}$ & $17.350(4)$ & [9] \\
\hline $\begin{array}{l}\mathrm{Tm}_{2} \mathrm{~S}_{3}(\mathrm{~F}) \\
1873 \mathrm{~K}, 1.0 \mathrm{GPa}\end{array}$ & $m P 20, P 2_{1} / m, \mathrm{Tm}_{2} \mathrm{~S}_{3}$ & $11.110(5)$ & $\begin{array}{l}3.874(3) \\
\beta=108.88^{\circ}\end{array}$ & $10.872(5)$ & {$[11]$} \\
\hline $1123 \mathrm{~K}, \mathrm{NaCl}$-flux & & $11.162(4)$ & $\begin{array}{l}3.9024(8) \\
\beta=108.87^{\circ}\end{array}$ & $10.897(3)$ & {$[12]$} \\
\hline
\end{tabular}

Table 2 Experimental and crystallographic data for $\delta-\mathrm{Tm}_{2} \mathrm{~S}_{3}$.

\begin{tabular}{l|l}
\hline Compound & $\mathrm{Tm}_{2} \mathrm{~S}_{3}(\delta$-form $)$ \\
Structure type & $\delta-\mathrm{Ho}_{2} \mathrm{~S}_{3}$ \\
Space group & $P 2_{1} / m$ \\
Z, Pearson symbol & $6, m P 30$ \\
Lattice parameters, $\AA$ & $a=10.0626(14) \quad b=3.9675(5) \quad c=17.396(2), \beta=98.655(11)^{\circ}$ \\
Volume V, $\AA^{3}$ & $686.60(16)$ \\
Crystal size, mm & $0.27 \times 0.07 \times 0.03$ \\
Crystal color & semitransparent metallic light grey \\
Absorption coefficient, $\mathrm{mm}^{-1}$ & 39.670 \\
Absorption correction & analytical, $T_{\min }=0.051, T_{\max }=0.305$ \\
Theta range for data collection & 4.09 to $25.30^{\circ}$ \\
Limiting indices & $-12 \leq h \leq 12,-4 \leq k \leq 3,-20 \leq l \leq 20$ \\
Reflections collected / unique & $3791 / 1425[\mathrm{R}(\mathrm{int})=0.0358]$ \\
Completeness to theta & $97.9 \%$ \\
Refinement method & full-matrix least-squares on $\mathrm{F}^{2}$ \\
Data / restraints / parameters & $1425 / 0 / 92$ \\
Goodness-of-fit on $\mathrm{F}^{2}$ & 1.243 \\
Final R indices [I 2sigma(I)] & $\mathrm{R} 1=0.0425$, wR2 $=0.0834$ \\
$\mathrm{R}$ indices (all data) & $\mathrm{R} 1=0.0445, \mathrm{wR} 2=0.0841$ \\
Extinction coefficient & $0.00024(7)$ \\
Largest diff. peak and hole & 2.676 and -1.775 e $\AA^{-3}$ \\
\hline
\end{tabular}

\section{Results and discussion}

The structure solution of $\mathrm{Tm}_{2} \mathrm{~S}_{3}$ by direct methods in space group $P 2_{1} / m$ confirmed the $\mathrm{Ho}_{2} \mathrm{~S}_{3}$ structure type [3]. The atomic parameters were refined in the anisotropic approximation down to $\mathrm{R}=0.0425$ and the final values are listed in Table 3 . Refinements of the occupancies of the cation sites indicated no deviation from full occupation by Tm. The cell parameters are in good agreement with those reported in the literature and the presence of $\mathrm{Ni}$ in the structure could be excluded. Fig. 1 shows a clinographic projection of the unit cell content of $\delta-\mathrm{Tm}_{2} \mathrm{~S}_{3}$ with indication of atomic connections Tm-S.

A look at the interatomic distances in Table 4 confirms the iono-covalent character of the chemical bonding in $\mathrm{Tm}_{2} \mathrm{~S}_{3}$. Some distances, e.g. the shortest distances Tm6-S9 in the $\left[\mathrm{TmS}_{6}\right]$ octahedron (2.594(5) $\AA$ ) are slightly shorter than the sum of ionic radii of $\mathrm{Tm}^{3-}$ and $\mathrm{S}^{2-}(2.79 \AA)$. 
J Kończyk et al., Crystal structure of $\delta-\mathrm{Tm}_{2} \mathrm{~S}_{3}$

Table 3 Atomic positional and displacement parameters for $\delta-\mathrm{Tm}_{2} \mathrm{~S}_{3}$ (all atoms in Wyckoff site $2 e: x^{1 / 4} \mathrm{z}$ with site occupation number $G=1$ ).

\begin{tabular}{|c|c|c|c|c|c|c|c|}
\hline \multirow[t]{2}{*}{ Atom } & \multirow[t]{2}{*}{$x$} & \multirow[t]{2}{*}{$z$} & \multicolumn{5}{|c|}{ Displacement parameters $^{\mathrm{a}}, \AA^{2} \times 10^{3}$} \\
\hline & & & $U_{\text {eq }}$ & $U_{11}$ & $U_{22}$ & $U_{33}$ & $U_{13}$ \\
\hline Tm1 & $0.1248(1)$ & $0.9299(1)$ & $7(1)$ & $9(1)$ & $7(1)$ & $5(1)$ & $1(1)$ \\
\hline Tm2 & $0.1867(1)$ & $0.2203(1)$ & $8(1)$ & $10(1)$ & $8(1)$ & $5(1)$ & $-1(1)$ \\
\hline $\mathrm{Tm} 3$ & $0.1891(1)$ & $0.5207(1)$ & $7(1)$ & $8(1)$ & $6(1)$ & $6(1)$ & $0(1)$ \\
\hline Tm4 & $0.4872(1)$ & $0.8989(1)$ & $9(1)$ & $11(1)$ & $7(1)$ & $8(1)$ & $3(1)$ \\
\hline Tm5 & $0.5490(1)$ & $0.6153(1)$ & $7(1)$ & $9(1)$ & $7(1)$ & $5(1)$ & $0(1)$ \\
\hline Tm6 & $0.8295(1)$ & $0.2813(1)$ & $8(1)$ & $12(1)$ & $7(1)$ & $4(1)$ & $0(1)$ \\
\hline $\mathrm{S} 1$ & $0.0083(5)$ & $0.7678(3)$ & $6(1)$ & $6(2)$ & $9(2)$ & $4(2)$ & $0(2)$ \\
\hline $\mathrm{S} 2$ & $0.0526(5)$ & $0.0751(3)$ & $7(1)$ & $8(2)$ & $11(3)$ & $3(2)$ & $2(2)$ \\
\hline S3 & $0.2680(5)$ & $0.3729(3)$ & $8(1)$ & $10(2)$ & $11(2)$ & $2(2)$ & $0(2)$ \\
\hline $\mathrm{S} 4$ & $0.3200(5)$ & $0.6826(3)$ & $8(1)$ & $10(2)$ & $3(2)$ & $13(3)$ & $4(2)$ \\
\hline S5 & $0.3668(5)$ & $0.0312(3)$ & $8(1)$ & $10(2)$ & $4(2)$ & $7(3)$ & $0(2)$ \\
\hline S6 & $0.6157(5)$ & $0.4673(3)$ & $5(1)$ & $6(2)$ & $4(2)$ & $5(2)$ & $-1(2)$ \\
\hline S7 & $0.6375(5)$ & $0.7825(3)$ & $9(1)$ & $10(2)$ & $10(2)$ & $4(2)$ & $-3(2)$ \\
\hline S8 & $0.7101(5)$ & $0.1315(3)$ & $8(1)$ & $10(2)$ & $6(2)$ & $8(3)$ & $0(2)$ \\
\hline S9 & $0.9516(5)$ & $0.4237(3)$ & $10(1)$ & $9(2)$ & $11(3)$ & $10(3)$ & $0(2)$ \\
\hline
\end{tabular}

${ }^{\mathrm{a}} U_{\mathrm{eq}}$ is defined as one third of the trace of the orthogonalized $U_{\mathrm{ij}}$ tensor.

The anisotropic displacement factor exponent takes the form: $-2 \pi^{2}\left[h^{2} \mathbf{a}^{* 2} U_{11}+\ldots+2 h k \mathbf{a} * \mathbf{b}^{*} U_{12}\right], U_{12}=U_{23}=0$.

Table 4 Interatomic distances $(\delta, \AA)$ and coordination numbers (C.N.) for $\delta$ - $\operatorname{Tm}_{2} \mathrm{~S}_{3}$.

\begin{tabular}{|c|c|c|c|c|c|c|c|}
\hline \multicolumn{2}{|c|}{ Atoms } & $\delta, \AA$ & C.N. & \multicolumn{2}{|c|}{ Atoms } & $\delta, \AA$ & C.N. \\
\hline \multirow[t]{5}{*}{ Tm1 } & $-2 \mathrm{~S} 2$ & $2.661(3)$ & 7 & S1 & $-2 \operatorname{Tm} 6$ & $2.783(3)$ & 5 \\
\hline & $-\mathrm{S} 2$ & $2.731(5)$ & & & $-2 \mathrm{Tm} 2$ & $2.819(4)$ & \\
\hline & $-\mathrm{S} 5$ & $2.783(5)$ & & & $-\mathrm{Tm} 1$ & $2.887(5)$ & \\
\hline & $-\mathrm{S} 1$ & $2.887(5)$ & & $\mathrm{S} 2$ & $-2 \mathrm{Tm} 1$ & $2.661(3)$ & 4 \\
\hline & $-2 \mathrm{~S} 8$ & $2.893(4)$ & & & $-\mathrm{Tm} 2$ & $2.679(5)$ & \\
\hline \multirow[t]{4}{*}{$\operatorname{Tm} 2$} & $-\mathrm{S} 3$ & $2.657(5)$ & 6 & & $-\mathrm{Tm} 1$ & $2.731(5)$ & \\
\hline & $-2 S 7$ & $2.663(4)$ & & S3 & $-\operatorname{Tm} 2$ & $2.658(5)$ & 4 \\
\hline & $-\mathrm{S} 2$ & $2.679(5)$ & & & $-2 \mathrm{Tm} 5$ & $2.694(4)$ & \\
\hline & $-2 \mathrm{~S} 1$ & $2.819(4)$ & & & $-\operatorname{Tm} 3$ & $2.803(5)$ & \\
\hline \multirow[t]{5}{*}{$\operatorname{Tm} 3$} & $-2 S 9$ & $2.700(4)$ & 7 & S4 & $-2 \operatorname{Tm} 6$ & $2.623(3)$ & 4 \\
\hline & $-\mathrm{S} 9$ & $2.710(5)$ & & & $-\mathrm{Tm} 5$ & $2.737(5)$ & \\
\hline & $-2 S 6$ & $2.777(4)$ & & & $-\operatorname{Tm} 3$ & $2.925(6)$ & \\
\hline & $-\mathrm{S} 3$ & $2.803(5)$ & & S5 & $-2 \mathrm{Tm} 4$ & $2.652(4)$ & 4 \\
\hline & $-\mathrm{S} 4$ & $2.925(6)$ & & & $-\mathrm{Tm} 4$ & $2.756(5)$ & \\
\hline \multirow[t]{4}{*}{ Tm4 } & $-2 \mathrm{~S} 5$ & $2.652(4)$ & 6 & & $-\mathrm{Tm} 1$ & $2.783(5)$ & \\
\hline & $-\mathrm{S} 7$ & $2.704(5)$ & & S6 & $-\operatorname{Tm} 5$ & $2.757(5)$ & 5 \\
\hline & $-\mathrm{S} 7$ & $2.756(5)$ & & & $-2 \operatorname{Tm} 3$ & $2.777(4)$ & \\
\hline & $-2 S 8$ & $2.800(4)$ & & & $-2 \operatorname{Tm} 5$ & $2.834(4)$ & \\
\hline \multirow[t]{5}{*}{ Tm5 } & $-2 \mathrm{~S} 3$ & $2.694(4)$ & 7 & S7 & $-2 \mathrm{Tm} 2$ & $2.663(4)$ & 4 \\
\hline & $-\mathrm{S} 4$ & $2.737(5)$ & & & $-\mathrm{Tm} 4$ & $2.704(5)$ & \\
\hline & $-\mathrm{S} 6$ & $2.757(5)$ & & & $-\mathrm{Tm} 5$ & $2.911(5)$ & \\
\hline & $-2 S 6$ & $2.834(4)$ & & $\mathrm{S} 8$ & $-\mathrm{Tm} 6$ & $2.701(5)$ & 5 \\
\hline & $-\mathrm{S} 7$ & $2.911(5)$ & & & $-2 \mathrm{Tm} 4$ & $2.800(4)$ & \\
\hline \multirow[t]{4}{*}{ Tm6 } & $-\mathrm{S} 9$ & $2.594(5)$ & 6 & & $-2 \mathrm{Tm} 1$ & $2.893(4)$ & \\
\hline & $-2 S 4$ & $2.623(3)$ & & S9 & $-\operatorname{Tm} 6$ & $2.594(6)$ & 4 \\
\hline & $-\mathrm{S} 8$ & $2.701(5)$ & & & $-2 \mathrm{Tm} 3$ & $2.700(4)$ & \\
\hline & $-2 \mathrm{~S} 1$ & $2.783(3)$ & & & $-\operatorname{Tm} 3$ & $2.710(5)$ & \\
\hline
\end{tabular}




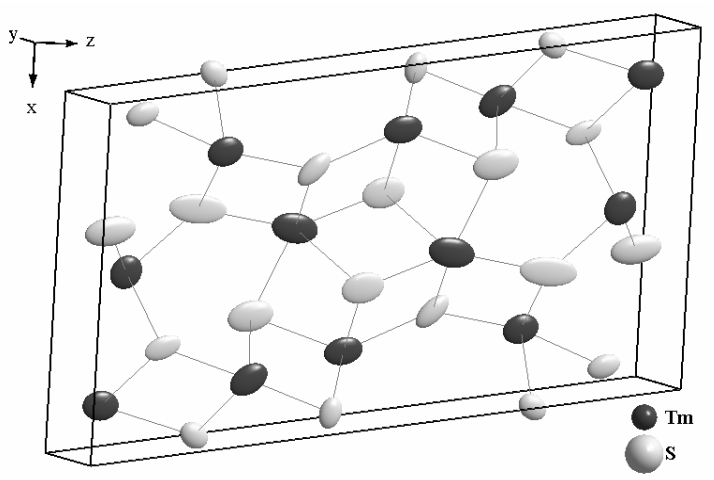

Fig. 1 Clinographic projection of the unit cell of $\delta-\mathrm{Tm}_{2} \mathrm{~S}_{3}$ with displacement ellipsoids and atomic connections of $\mathrm{Tm}$ and $\mathrm{S}$ atoms between layers with $y=1 / 4$ and $y=3 / 4$.

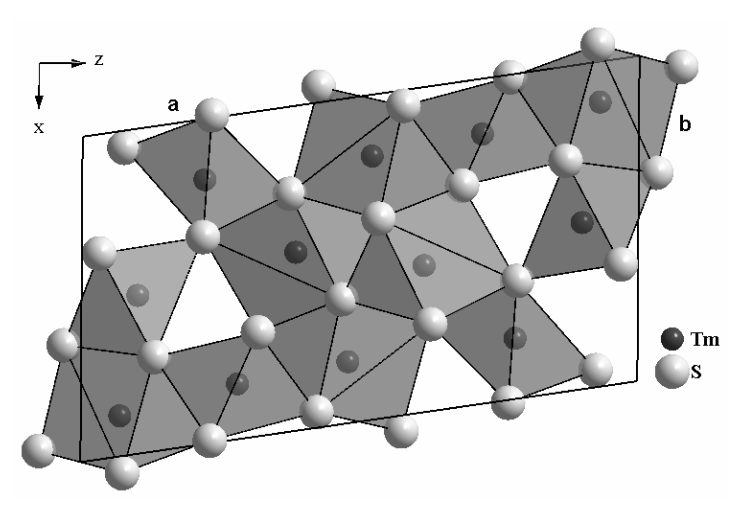

Fig. 2 Stacking of distorted octahedrons $\left[\mathrm{TmS}_{6}\right]$ (a) and mono-capped trigonal prisms $\left[\mathrm{TmS}_{7}\right]$ (b) in $\delta-\mathrm{Tm}_{2} \mathrm{~S}_{3}$.

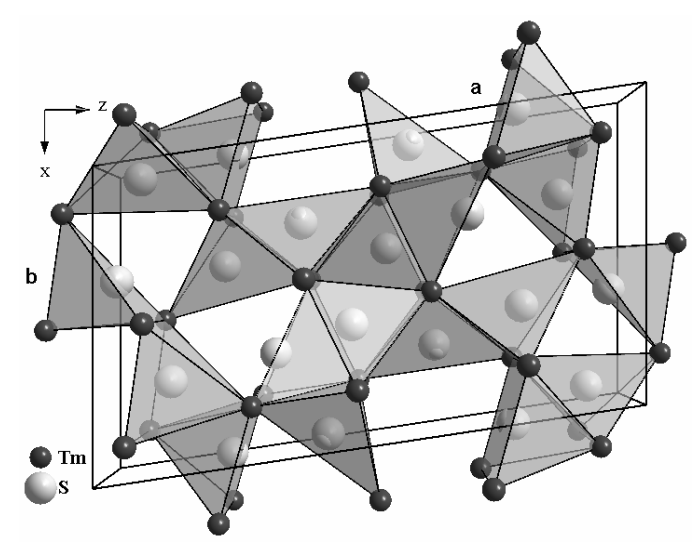

Fig. 3 Stacking of tetragonal pyramids [STm ${ }_{5}$ ] (a) and trigonal pyramids $\left[\mathrm{STm}_{4}\right]$ (b) in $\delta-\mathrm{Tm}_{2} \mathrm{~S}_{3}$. The sulfur atoms are situated in the bases of the pyramids.

For half of the thulium atoms the coordination number is 6 . The interatomic distances within the considerably distorted octahedra range from 2.59 to
$2.82 \AA$. The remaining thulium atoms have coordination number 7 , with $\mathrm{Tm}-\mathrm{S}$ distances between $\sim 2.7$ and $2.9 \AA$ A The coordination polyhedra can for all three crystallographically independent sites be assimilated to a mono-capped trigonal prism $\left[\mathrm{TmS}_{7}\right]$. The trigonal prisms, as well as the octahedra, share edges to form infinite columns in the direction of the unique axis of the monoclinic structure. Fig. 2 shows the stacking of these polyhedra, which share atoms to form a 3D-framework. Fig. 3 shows the same structure, but focusing this time on the environment of the sulfur atoms. Three of the sulfur atom sites are surrounded by five cations forming tetragonal pyramids $\left[\mathrm{STm}_{5}\right]$, while for the other sulfur atoms the coordination number is 4 and the coordination polyhedra are distorted tetrahedra $\left[\mathrm{STm}_{4}\right]$.

\section{References}

[1] E.I. Yarembash, A.A. Eliseev, Chalcogenides of rare-earth metals, Akad. Nauk SSSR, Nauka, Moscow, 1975 (in Russian).

[2] A.A. Eliseev, G.M. Kuzmichyeva, in: K.A. Gschneidner, Jr, L. Eyring (Eds.), Handbook on the Physics and Chemistry of Rare Earths, Vol. 13, North-Holland, Amsterdam, 1990, Ch. 89, pp. 191-281.

[3] J.G. White, P.N. Yocom, S. Lerner, Inorg. Chem. 6 (1967) 1872-1875.

[4] A.W. Sleight, C.T. Prewitt, Inorg. Chem. 7 (1968) 2282.

[5] A.A. Grizik, A.A. Eliseev, G.P. Borodulenko, G.M. Kuzmicheva, V.A. Tolstova, Zh. Neorg. Khim. 21 (1976) 3208-3212.

[6] K.-J. Range, H. Drexler, A. Gietl, U. Klement, K.G. Lange, Acta Crystallogr. C 46 (1990) 487488.

[7] A.A. Eliseev, A.A. Grizik, G.M. Kuzmichyeva, G.P. Borodulenko, Russ. J. Inorg. Chem. 20(7) (1975) 973-974.

[8] G.M. Kuzmichyeva, E.I. Smarina, S.Yu. Khlyustova, V.V. Chernyshev, Russ. J. Inorg. Chem. 35(4) (1990) 488-490.

[9] K.-J. Range, A. Gietl, U. Klement, K.G. Lange, J. Less-Common Met. 158 (1990) L21-L25.

[10] K.-J. Range, R. Leeb, Z. Naturforsch. B 30 (1975) 889-895.

[11] K.-J. Range, R. Leeb, Z. Naturforsch. B 31 (1976) 311-314.

[12] T. Schleid, F. Lissner, J. Alloys Compd. 189 (1992) 69-74.

[13] Oxford Diffraction, CrysAlis CCD. Version 1.170, Oxford Diffraction Ltd., Abingdon, Oxford, England, 2003.

[14] Oxford Diffraction, CrysAlis RED. Version 1.171, Oxford Diffraction Ltd., Abingdon, Oxford, England, 2005. 
J Kończyk et al., Crystal structure of $\delta-\mathrm{Tm}_{2} \mathrm{~S}_{3}$

[15] G. M. Sheldrick, SHELXS97 and SHELXL97 WinGX Version, Release 97-2, University of Göttingen, Germany, 1997.
[16] L.M. Gelato, E. Parthé, J. Appl. Crystallogr. 20 (1987) 139-143.

Proceeding of the IX International Conference on Crystal Chemistry of Intermetallic Compounds, Lviv, September 20-24, 2005. 